\section{A influência das doenças crônicas na capacidade funcional dos idosos do Município de São Paulo, Brasil}

\author{
The effect of chronic diseases on functional status \\ of the elderly living in the city of São Paulo, Brazil
}

\begin{abstract}
The main focus of this study was the effect of chronic disease (hypertension, diabetes mellitus, heart disease, lung disease, cancer, and arthropathy) on the functional status (activities of daily living - ADL, instrumental activities of daily living - IADL) among the elderly, controlling for age, gender, living arrangements, education, and comorbidity. The analysis was based on information provided by the SABE Project, from the city of São Paulo, Brazil, including individuals 60 years of age and over ( $n=1,769)$, from January 2000 to March 2001. A multinomial logistic regression model was used. Compared to the absence of dependency category, heart disease $(O R=1.82)$, arthropathy $(O R=1.59)$, lung disease $(O R=1.50)$, and hypertension $(O R=1.39)$ were the main diseases that affected the IADL dependency category. Lung disease (OR $=2.58)$, arthropathy $(O R=2.27)$, hypertension $(O R=$ 2.13), and heart disease ( $O R=2.10)$ had important impact on the IADL and ADL dependency categories. The results were statistically significant $(p<0.05)$.
\end{abstract}

Aged; Chronic Diseases; Motor Activity
Luciana Correia Alves 1

Beatriz Consuelo Quinet Leimann ${ }^{1}$

Maria Estrella López Vasconcelos 1

Marilia Sá Carvalho ${ }^{1}$

Ana Glória Godoi Vasconcelos 1

Thaís Cristina Oliveira da Fonseca 2

Maria Lúcia Lebrão ${ }^{3}$

Ruy Laurenti 3

\section{Introdução}

Desde as últimas décadas do século passado, o Brasil se depara com um declínio rápido e acentuado da fecundidade, fenômeno sem precedentes na sua história, e que se sobressai mesmo em comparação com outros países, seja do mundo desenvolvido, seja entre aqueles em desenvolvimento. Como aconteceu na maioria destes países, esse declínio, combinado com a queda da mortalidade, acarretou um processo de envelhecimento populacional e de aumento da longevidade da população.

Apesar do processo de envelhecimento não estar, necessariamente, relacionado a doenças e incapacidades, as doenças crônico-degenerativas são freqüentemente encontradas entre os idosos 1. Assim, a tendência atual é termos um número crescente de indivíduos idosos que, apesar de viverem mais, apresentam maiores condições crônicas. E o aumento no número de doenças crônicas está diretamente relacionado com maior incapacidade funcional.

O comprometimento da capacidade funcional do idoso tem implicações importantes para a família, a comunidade, para o sistema de saúde e para a vida do próprio idoso, uma vez que a incapacidade ocasiona maior vulnerabilidade e dependência na velhice, contribuindo para a diminuição do bem-estar e da qualidade de vida dos idosos. 
Deste modo, a capacidade funcional surge como um novo componente no modelo de saúde dos idosos 2 e particularmente útil no contexto do envelhecimento, porque envelhecer mantendo todas as funções não significa problema para o indivíduo ou sociedade. O problema se inicia quando as funções começam a deteriorar 3 .

A incapacidade funcional pode ser definida como a inabilidade ou a dificuldade de realizar tarefas que fazem parte do cotidiano do ser humano e que normalmente são indispensáveis para uma vida independente na comunidade 4 . Por sua vez, a capacidade funcional se refere à potencialidade para desempenhar as atividades de vida diária ou para realizar determinado ato sem necessidade de ajuda 5 , imprescindíveis para proporcionar uma melhor qualidade de vida.

As atividades de vida diária (AVDs), as atividades instrumentais de vida diária (AIVDs) e mobilidade são as medidas freqüentemente utilizadas para avaliar a capacidade funcional do indivíduo. As AVDs consistem nas tarefas de auto-cuidado, como tomar banho, vestir-se e alimentar-se e se baseiam no índice de Katz ${ }^{6}$. Essa medida reflete um substancial grau de incapacidade. Em geral, quanto maior o número de dificuldades que uma pessoa tem com as AVDs, mais severa é a sua incapacidade. A prevalência de dificuldade ou necessidade de ajuda em realizar AVDs é inferior à prevalência das demais medidas de incapacidade funcional.

As AIVDs foram as escalas desenvolvidas por Lawton \& Brody 7 em 1969 e indicam tarefas mais adaptativas ou necessárias para vida independente na comunidade, como, por exemplo, fazer compras, telefonar, utilizar o transporte, realizar tarefas domésticas, preparar uma refeição, cuidar do próprio dinheiro. Essas tarefas são consideradas mais difíceis e complexas do que as AVDs.

A mobilidade constitui um outro componente essencial da avaliação funcional. Refere-se à capacidade de sair da residência e pode ser mensurada por meio de um enfoque hierárquico, iniciando-se com tarefas simples como transferir-se da cama para a cadeira e progredindo para as tarefas mais complexas como caminhadas de curtas e longas distâncias, subir e descer escadas, atividades que exigem amplitude de movimento, resistência e força muscular 8 . Essa tarefa possui um grau de complexidade intermediária entre as AVDs e as AIVDs. Sendo assim, alguns autores 9,10 propõem operacionalizar a incapacidade por meio de uma escala de incapacidade funcional hierárquica que, em geral, relaciona os indicadores de AVD, AIVD e mobilidade distinguindo quatro categorias: independente, dependente somente nas AIVDs, dependente nas AIVDs e mobilidade, dependente nas AIVDs, mobilidade e AVDs.

Estudos demonstram que fatores sócio-demográficos como idade, sexo, arranjo familiar e educação têm influência sobre a capacidade funcional do idoso 11,12.

Diversos estudos demonstram associações importantes entre doenças crônicas e incapacidade funcional dos idosos 13,14,15,16. As diversas condições crônicas existentes não possuem impactos similares na funcionalidade. Em um idoso que apresente várias afecções crônicas, a incapacidade de realização de muitas ou de todas as atividades pode dever-se ao efeito de uma única condição de morbidade ou ao efeito independente de várias condições, cada uma delas afetando somente determinadas atividades 17 . Poucos pesquisadores têm se preocupado em investigar o efeito de uma doença crônica específica na capacidade funcional dos idosos, ou seja, o impacto independente de cada doença.

Compreender os fatores que contribuem para a incapacidade funcional do idoso pode auxiliar os planejadores de políticas públicas na (re)organização de estratégias preventivas mais eficazes que irão, conseqüentemente, reduzir os custos com o serviço de saúde e minimizar a carga sobre a família.

O objetivo principal deste estudo foi investigar a influência das doenças crônicas na capacidade funcional dos idosos residentes no Município de São Paulo nos anos de 2000/2001, controlando por idade, sexo, arranjo familiar, educação e presença de outras comorbidades. Objetivamos avaliar o efeito independente de cada uma das seis doenças crônicas (hipertensão arterial, diabetes mellitus, doença cardíaca, doença pulmonar, câncer e artropatia).

\section{Material e métodos}

O estudo foi desenvolvido com base em dados oriundos do Projeto Saúde, Bem-estar e Envelhecimento na América Latina e Caribe (Projeto $\mathrm{SABE}$ ), que constitui um estudo epidemiológico transversal, de base populacional domiciliar, sob a coordenação da Organização Pan-Americana da Saúde (OPAS). O objetivo desse projeto é investigar os diversos aspectos referentes à saúde da população idosa residente em área urbana de sete países da América Latina e Caribe: Argentina, Barbados, Brasil, Chile, Cuba, México e Uruguai 18. No Brasil, o Projeto SABE circunscreveuse aos limites territoriais do Município de São Paulo. Foram entrevistados 2.143 idosos (indivíduos com 60 anos e mais), não institucionalizados, de ambos os sexos, no período entre janeiro 
de 2000 e março de 2001. O processo de amostragem foi realizado em dois estágios. A base de dados contém informações sobre: características demográficas, sociais, econômicas, da família e do domicílio, das condições crônicas e agudas de saúde, do estado funcional, do estado cognitivo, do uso de medicamentos, do estado nutricional, uso e acesso a serviços de saúde, transferências familiares e institucionais, rede de apoio social, fontes de renda, história ocupacional e aposentadoria 19 .

No presente estudo foram selecionados todos os indivíduos com 60 anos e mais de idade, de ambos os sexos, sem alteração cognitiva. Assim, a amostra considerada é constituída de 1.769 idosos. Para avaliação do estado cognitivo do idoso foi utilizada a versão abreviada do Mini-Mental State Examination de Folstein (MMSE), conhecida como AMMSE. Os indivíduos que alcançaram um escore 12 ou menos foram classificados como tendo déficit cognitivo e, portanto, excluídos da amostra.

A variável dependente é a capacidade funcional do idoso. Para mensurá-la foram utilizadas as informações das AVDs: atravessar um quarto caminhando, alimentar-se, deitar e levantar da cama, usar o vaso sanitário, vestir e despir, tomar banho; e das AIVDs: preparar uma refeição quente, cuidar do próprio dinheiro, usar um transporte, fazer compras, telefonar, fazer tarefas domésticas leves, fazer tarefas domésticas pesadas, tomar medicamento. No questionário do Projeto SABE os participantes foram interrogados sobre a presença de dificuldades ou necessidade de ajuda com cada atividade. Os idosos foram classificados como independentes caso não relatassem dificuldade para realizar nenhuma AVD e AIVD, e dependentes quando relataram necessidade de ajuda em pelo menos uma das atividades de cada dimensão. Conforme proposto por Hoyemans et al. 9, uma escala de incapacidade funcional hierárquica foi construída distinguindo três categorias: independentes, dependentes nas AIVDs, dependentes nas AVDs e AIVDs.

As variáveis independentes foram divididas em sócio-demográficas e em enfermidades crônicas. As seguintes variáveis sócio-demográficas foram consideradas para controle: idade, sexo, arranjo familiar e educação. A idade foi avaliada como variável contínua. O arranjo familiar foi dicotomizado em: mora sozinho(a), mora acompanhado(a). Com relação ao nível educacional, os idosos foram categorizados em: sem escolaridade, um a quatro anos de estudos e cinco ou mais anos de estudo. As doenças crônicas foram mensuradas com base nas respostas afirmativas (auto-referidas) relativas à presença de seis doenças crônicas: hipertensão arterial, diabetes mellitus, doença cardíaca, doença pulmonar, câncer e artropatia. A doença cardíaca foi definida como ataque cardíaco, doença coronária, angina, doença congestiva ou outros problemas cardíacos. A doença pulmonar como asma, bronquite ou enfisema. A artropatia como artrite, reumatismo ou artrose. O câncer excluía somente os tumores menores de pele.

Para estimar a associação entre a escala de incapacidade funcional hierárquica com as doenças crônicas, controlando pelas variáveis sócio-demográficas e pelas demais enfermidades crônicas foi realizada uma análise de regressão logística multinomial múltipla.

O modelo de regressão logístico multinomial é definido por:

$\ln \left(\frac{p_{i k}}{p_{i 3}}\right)=\beta_{0 k}+\beta_{1 k} x_{i 1}+\beta_{2 k} x_{i 2}+\ldots+\beta_{p k} x_{i p}, k=1,2$

No modelo multinomial o número de equações é igual ao número de categorias da variável dependente menos 1 , ou seja, K-1. No presente estudo, a variável resposta possui três categorias. As categorias dependente nas AIVDs e dependente nas AIVDs e AVDs da variável-reposta foram comparadas em relação à categoria independente.

Os resultados do modelo foram apresentados como razões de chance (odds ratio) e os respectivos intervalos de confiança de $95 \%$. As análises dos dados foram realizadas na biblioteca nnet do R versão 2.2.0 (R Development Core Team: http://www.R-project.com).

\section{Resultados}

A idade dos idosos variou de 60 a 96 anos, sendo que as idades média e mediana foram 71,93 e 72 anos, respectivamente ( $\mathrm{DP}=7,79$ ). A população era predominantemente feminina $(58,8 \%)$. A hipertensão arterial foi a condição crônica mais freqüente $(53,4 \%)$, seguida por artropatia $(33,8 \%)$, doença cardíaca $(20,6 \%)$, diabetes mellitus $(17,5 \%)$ doença pulmonar $(12,5 \%)$ e câncer $(3,6 \%)$. A presença de doença cardíaca $(32,2 \%)$, artropatia (30,9\%), câncer (30\%) e doença pulmonar $(27,2 \%)$ foi encontrada em maior proporção entre os idosos dependentes nas AIVDs. Os idosos dependentes nas AIVDs e AVDs apresentavam maior prevalência de doença pulmonar (10\%), seguida da doença cardíaca $(8,5 \%)$ e artropatia $(7,5 \%)$.

A Tabela 1 mostra os resultados da regressão logística multinomial múltipla. Com relação à resposta dependente nas AIVDs em comparação à categoria de referência independente, observase que depois de ajustar por idade, sexo, arranjo 
Razões de chances (OR) ajustadas por idade, sexo, arranjo familiar e escolaridade, tendo como variável resposta dependente nas atividades instrumentais de vida diária (AIVDs) e dependente nas atividades de vida diária (AVDs) e AIVDs em relação à categoria independente.

\begin{tabular}{|c|c|c|c|c|c|c|}
\hline \multirow[t]{2}{*}{ Variável } & \multicolumn{3}{|c|}{ Dependente nas AIVDs } & \multicolumn{3}{|c|}{ Dependente nas AIVDs e AVDs } \\
\hline & OR (IC95\%) & p-valor & $\mathrm{n}$ & OR (IC95\%) & p-valor & $\mathrm{n}$ \\
\hline \multicolumn{7}{|c|}{ Hipertensão arterial } \\
\hline Não & 1,00 & & 148 & 1,00 & & 25 \\
\hline Sim & $1,39(1,08-1,80)$ & 0,01 & 256 & $2,13(1,28-3,56)$ & 0,00 & 61 \\
\hline \multicolumn{7}{|c|}{ Diabetes mellitus } \\
\hline Não & 1,00 & & 323 & 1,00 & & 65 \\
\hline Sim & $1,25(0,91-1,71)$ & 0,17 & 181 & $1,57(0,91-2,71)$ & 0,11 & 21 \\
\hline \multicolumn{7}{|l|}{ Câncer } \\
\hline Não & 1,00 & & 385 & 1,00 & & 82 \\
\hline Sim & $1,24(0,67-2,30)$ & 0,49 & 19 & $1,40(0,46-4,26)$ & 0,55 & 4 \\
\hline \multicolumn{7}{|c|}{ Doença cardíaca } \\
\hline Não & 1,00 & & 285 & 1,00 & & 55 \\
\hline Sim & $1,82(1,37-2,42)$ & 0,00 & 119 & $2,10(1,28-3,43)$ & 0,00 & 31 \\
\hline \multicolumn{7}{|c|}{ Artropatia } \\
\hline Não & 1,00 & & 219 & 1,00 & & 41 \\
\hline Sim & $1,59(1,24-2,05)$ & 0,00 & 185 & $2,27(1,42-3,65)$ & 0.00 & 45 \\
\hline \multicolumn{7}{|c|}{ Doença pulmonar } \\
\hline Não & 1,00 & & 344 & 1,00 & & 64 \\
\hline Sim & $1,50(1,05-2,15)$ & 0,03 & 60 & $2,58(1,49-4,48)$ & 0,00 & 22 \\
\hline
\end{tabular}

Fonte dos dados básicos: Projeto SABE (2000/2001).

familiar, escolaridade e demais doenças crônicas, as doenças que exercem uma significativa influência na dependência funcional do idoso são a doença cardíaca $(\mathrm{OR}=1,82)$, a artropatia $(\mathrm{OR}=1,59)$, a doença pulmonar $(\mathrm{OR}=1,50)$ e a hipertensão arterial $(\mathrm{OR}=1,39)$. Diabetes mellitus e câncer não apresentam associação estatisticamente significativa $(\mathrm{p}>0,05)$. Quanto à resposta dependente nas AIVDs e AVDs em relação à categoria de referência independente, os resultados mostram que a doença pulmonar $(\mathrm{OR}=2,58)$, a artropatia $(\mathrm{OR}=2,27)$, a hipertensão arterial (OR $=2,13$ ) e a doença cardíaca $(\mathrm{OR}=2,10)$ demonstram uma forte associação com a dependência nas AIVDs e AVDs. Os resultados são estatisticamente significativos $(\mathrm{p}<0,05)$. Diabetes mellitus e câncer não são relacionados de forma estatisticamente significativa com a dependência nas AIVDs e AVDs.

\section{Discussão}

A vantagem do Projeto SABE em relação às outras pesquisas que abordam as condições de saúde no Brasil é que além de ser um estudo específico de saúde direcionado exclusivamente aos indiví- duos de 60 anos e mais, ele contém informações bem coletadas sobre vários domínios da saúde e de fatores diretamente relacionados a ela.

Existem diversas possibilidades de avaliar o efeito de doenças crônicas sobre a capacidade funcional do idoso. Optamos por analisar o impacto de diferentes doenças isoladamente considerando que algumas dessas condições podem ter uma influência maior do que outras sobre a capacidade funcional do idoso, uma vez que, de acordo com Guccione et al. 17 não há razão para assumir que todas as condições apresentem impactos similares na função.

Os resultados deste estudo demonstraram que as doenças crônicas apresentam uma forte influência na capacidade funcional do idoso. A presença de hipertensão arterial aumenta em $39 \%$ a chance de o idoso ser dependente nas AIVDs, a doença cardíaca aumenta em $82 \%$, a artropatia em $59 \%$ e a doença pulmonar em $50 \%$. Para a dependência nas AIVDs e AVDs, a chance mais do que dobrou para a presença de cada uma dessas doenças crônicas.

A presença de câncer não ocasionou um impacto estatisticamente significativo na dependência funcional do idoso. Guccione et al. $17 \mathrm{e}$ Garman et al. 20 também não encontraram as- 
sociação entre incapacidade funcional e câncer. No idoso sobrevivente de câncer a presença de comorbidades mais do que a história de câncer em si estaria correlacionada com a incapacidade funcional ${ }^{20}$. Com relação ao diabetes mellitus, o presente estudo também não encontrou uma associação estatisticamente significativa com a capacidade funcional do idoso. A literatura mostra uma diminuição da capacidade funcional entre os idosos diabéticos 15,21. Rekeneire et al. 22 relatam uma associação entre diabetes mellitus e a presença de indicadores precoces de declínio funcional. Bayliss et al. 23 mostraram que a chance de o idoso apresentar um declínio da capacidade funcional foi duas vezes maior entre os diabéticos quando comparados com os indivíduos hipertensos. Uma possível explicação para a falta de associação entre diabetes mellitus e dependência funcional pode ser o fato de que a população de diabéticos esteja subestimada em nossa amostra. Torquato et al.24 observaram prevalência de diabetes mellitus em $21,7 \%$ dos indivíduos de 60 a 69 anos residentes em Ribeirão Preto. No nosso estudo a prevalência observada foi de $17,5 \%$. Também devemos considerar que a associação entre diabetes mellitus e incapacidade funcional é devida a múltiplos fatores porque esta doença está relacionada, sobretudo, a complicações vasculares e neuropáticas que, conseqüentemente, afetam a capacidade funcional. Sendo assim, o controle para outras comorbidades, como doença cardíaca, atenuaria a associação entre diabetes mellitus e incapacidade funcional, conforme observado por Gregg et al. 25 e Volpato et al. 26.

Verbrugge et al. 27 evidenciaram que a diabetes mellitus, as doenças cardíacas, a artrite e o câncer apresentam um moderado impacto; e a hipertensão arterial é a condição menos provável de ocasionar efeito na capacidade funcional do idoso. Guccione et al. 17 realizaram um estudo utilizando os dados do Framingham Study Cohort, com o objetivo de identificar associações entre condições crônicas e dependência funcional dos idosos americanos e encontraram que a artrite e a doença cardíaca apresentavam maiores efeitos em comparação com as outras doenças crônicas.

Em nosso estudo utilizamos os dados do Projeto SABE sobre a presença de doenças crônicas que se baseiam na informação auto-referida.
Segundo estudo de Wu et al. ${ }^{28}$, a morbidade auto-referida tende a subestimar a prevalência de doenças cardíacas (menos $21 \%$ ) e hipertensão (menos $4 \%$ ) e a superestimar ligeiramente a prevalência de diabetes mellitus (mais $2 \%$ ). Cox et al. 29 observaram correlação significativa entre lombalgia auto-referida e escala padronizada de performance. Assim, apesar das restrições da utilização da morbidade referida, acreditamos que nossos resultados sejam consistentes, sobretudo considerando que os dados poderiam estar subestimados e não superestimados.

Este estudo utilizou a regressão logística multinomial múltipla que tem a vantagem de desagregar a capacidade funcional em um número maior de categorias. Esta desagregação possibilitou a obtenção de resultados mais detalhados, em vários níveis da dependência funcional do idoso, ao invés de apenas nas categorias dependente e independente como na regressão logística. Alguns aspectos, entretanto, limitam o uso destes modelos. Em primeiro lugar, talvez fosse adequado considerar que a incapacidade funcional poderia ser ordenada e o risco estimado em relação à categoria anterior. Contudo, ainda que exista uma ordenação subjacente, a comparação dos tipos de incapacidade com a total capacidade permite avaliar o efeito das co-morbidades, sempre tendo a mesma linha de base de comparação. Além disso, estes modelos ainda são pouco usados em epidemiologia, e as rotinas de análise de ajuste e resíduos não estão totalmente desenvolvidas.

Cabe lembrar que a gravidade das doenças estudadas e o tratamento adotado podem ter influenciado as associações encontradas entre as doenças crônicas e a capacidade funcional. Entretanto, o Projeto SABE não contempla esse tipo de informação. Além disso, o agrupamento de diferentes patologias sob a mesma denominação implica diferentes chances de desenvolver incapacidade.

A manutenção da capacidade funcional pode ter implicações para a qualidade de vida dos idosos, por estar relacionada com a capacidade do indivíduo se manter na comunidade, desfrutando a sua independência até as idades mais avançadas. Os achados deste estudo sugerem que a prevenção e o controle das doenças crônicas podem melhorar as atividades e, conseqüentemente, promover o bem-estar desta população. 


\section{Resumo}

O objetivo principal deste estudo foi investigar a influência de doenças crônicas (hipertensão arterial, diabetes mellitus, doença cardíaca, doença pulmonar, câncer e artropatia) na capacidade funcional (atividades de vida diária-AVDs - e atividades instrumentais de vida diária - AIVDs) dos idosos, controlando por idade, sexo, arranjo familiar, educação e presença de outras comorbidades. Os dados foram obtidos do Projeto $S A B E$ que inclui pessoas de 60 anos e mais, residentes no Município de São Paulo, Brasil, entre janeiro de 2000 e março de 2001. A amostra foi constituída de 1.769 idosos. Para a análise dos dados foi utilizada a regressão logística multinomial múltipla. Em comparação com a categoria de referência independente, as doenças que exercem uma significativa influência na categoria dependente nas AIVDs são a doença cardíaca $(O R=1,82)$, a artropatia $(O R=1,59)$, a doença pulmonar $(O R=1,50)$ e a hipertensão arterial $(O R=1,39)$. Quanto à resposta na categoria dependente nas AIVDs e AVDs, os resultados mostram que a doença pulmonar $(O R=2,58)$, a artropatia $(O R=2,27)$, a hipertensão arterial $(O R=2,13)$ e a doença cardíaca $(O R=2,10)$ demonstram um forte efeito. Os resultados são estatisticamente significativos $(p<0,05)$.

Idoso; Doenças Crônicas; Atividade Motora

\section{Colaboradores}

L. C. Alves e B. C. Q. Leimann foram responsáveis pela revisão bibliográfica, análise dos dados, estruturação e redação do artigo. M. E. L. Vasconcelos participou da revisão bibliográfica e análise dos dados. M. S. Carvalho participou da discussão e da revisão do artigo. A. G. G. Vasconcelos participou da revisão do artigo. T. C. O. Fonseca participou da análise dos dados. M. L. Lebrão é co-investigador principal do Projeto SABE e participou da revisão do artigo. R. Laurenti é co-investigador principal do Projeto SABE.

\section{Agradecimentos}

Este trabalho contou com o apoio do Conselho Nacional de Desenvolvimento Científico e Tecnológico (CNPq). Os autores agradecem também a Organização Pan-Americana da Saúde. O Projeto SABE foi financiado pela Fundação de Amparo à Pesquisa do Estado de São Paulo (FAPESP).

\section{Referências}

1. Chaimowicz F. Os idosos brasileiros no século XXI. Belo Horizonte: Postgraduate; 1998.

2. Ramos LR. Fatores determinantes do envelhecimento saudável em idosos residentes em centro urbano: Projeto Epidoso, São Paulo. Cad Saúde Pública 2003; 19:793-7.

3. Kalache A, Veras RP, Ramos LR. O envelhecimento da população mundial. Um desafio novo. Rev Saúde Pública 1987; 21:200-10.

4. Yang Y, George LK. Functional disability, disability transitions, and depressive symptoms in late life. J Aging Health 2005; 17:263-92.

5. Farinati PTV. Avaliação da autonomia do idoso: definição de critérios para uma abordagem positiva a partir de um modelo de interação saúde-autonomia. Arq Geriatr Gerontol 1997; 1:1-9.

6. Katz S, Ford AB, Moskowitz RW, Jackson BA, Jaffe MW. Studies of illness in the aged. The index of ADL: a standardized measure of biological and psychosocial function. JAMA 1963; 185:914-9.

7. Lawton MP, Brody EM. Assessment of older people: self-maintaining and instrumental activities of daily living. Gerontologist 1969; 9:179-86.
8. Guralnik JM, Fried LP, Salive ME. Disability as a public health outcome in the aging population. Annu Rev Public Health 1996; 17:25-46.

9. Hoeymans N, Feskens EJM, van den Bos GAM, Kromhout D. Measuring functional status: crosssectional and longitudinal associations between performance and self-report (Zuthen Elderly Study 1990-1993). J Clin Epidemiol 1996; 49:1103-10.

10. Peres K, Verret C, Alioum A, Barberger-Gateau P. The disablement process: factors associated with progression of disability and recovery in French elderly people. Disabil Rehabil 2005; 27:263-76.

11. Koukouli S, Vlachonikolis IG, Philalithis A. Sociodemographic factors and self-reported functional status: the significance of social support. BMC Health Serv Res 2002; 2:20-34.

12. Parahyba MI, Veras R, Melzer D. Incapacidade funcional entre as mulheres idosas no Brasil. Rev Saúde Pública 2005; 39:383-91.

13. Rosa TEC, Benicio MHD, Latorre MRDO, Ramos LR. Fatores determinantes da capacidade funcional entre idosos. Rev Saúde Pública 2003; 37:40-8. 
14. Freedman VA, Martin LG. Contribution of chronic conditions to aggregate changes in old-age functioning. Am J Public Health 2000; 90:1755-60.

15. Bardage C, Isacson DG. Hypertension and healthrelated quality of life. An epidemiological study in Sweden. J Clin Epidemiol 2001; 54:172-81.

16. Kattainen A, Koskinen S, Reunanen A, Martelin T, Knekt P, Aromaa A. Impact of cardiovascular diseases on activity limitations and need for help among older persons. J Clin Epidemiol 2004; 57:828.

17. Guccione AA, Felson DT, Anderson JJ, Anthony JM, Zhang Y, Wilson PW, et al. The effects of specific medical conditions on the functional limitations of elders in the Framingham study. Am J Public Health 1994; 84:351-8.

18. Peláez M, Palloni A, Albala C, Alfonso JC, HamChande R, Hennis A, et al. Survey on aging, health and well-being, 2000. Washington DC: Pan American Health Organization/World Health Organization; 2003.

19. Lebrão ML, Duarte YAO. O projeto SABE no Brasil: uma abordagem inicial. Brasília: Organização PanAmericana da Saúde; 2003.

20. Garman KS, Pieper CF, Seo P, Cohen HJ. Function in elderly cancer survivors depends on comorbities. J Gerontol A Biol Sci Med Sci 2003; 58:M111924.

21. Wensing M, Vingerhoets E, Grol R. Functional status, health problems, age and comorbidity in primary care patients. Qual Life Res 2001; 10:141-8.

22. Rekeneire N, Resnick HE, Schwartz AV, Shorr RI, Kuller LH, Simonsick EM, et al. Diabetes is associated with subclinical functional limitation in nondisabled older individuals. Diabetes Care 2003; 26:3257-63.
23. Bayliss EA, Bayliss MS, Ware Jr. JE, Steiner JF. Predicting declines in physical function in persons with multiple chronic medical conditions: what we can learn from the medical problem list. Health Qual Life Outcomes 2004; 2:47-54.

24. Torquato MT, Montenegro Junior RM, Viana LA. Prevalence of diabetes mellitus and impaired glucose tolerance in the urban population aged 3069 years in Ribeirão Preto (São Paulo), Brazil. Sao Paulo Med J 2003; 121:224-30.

25. Gregg EW, Mangione CM, Cauley JA, Thompson TJ, Schwartz AV, Ensrud KE, et al. Diabetes and incidence of functional disability in older women. Diabetes Care 2002; 25:61-7.

26. Volpato S, Blaum C, Resnick H, Ferrucci L, Fried LP, Guralnik JM. Comorbidities and impairments explaining the association between diabetes and lower extremity disability. Diabetes Care 2002; 25:678-83.

27. Verbrugge LM, Lepkowski JM, Imanaka Y. Comorbidity and its impact on disability. Milbank Q 1989; 67:450-84.

28. Wu SC, Li CY, Ke DS. The agreement between self-reporting and clinical diagnosis for selected medical conditions among elderly in Taiwan. Publ Health 2000; 114:137-42.

29. Cox ME, Asselin S, Gracovestsky SA, Richards MP, Newman NM, Karakusevic V, et al. Relationship between functional evaluation measures and self-assessment in non-acute low back pain. Spine 2000; 25:1817-26.

Recebido em 23/Jun/2006

Versão final reapresentada em 02/Fev/2007

Aprovado em 07/Fev/2007 